\title{
The Association of Quantitative Facial Color Features with Cold Pattern in Traditional East Asian Medicine
}

\author{
Sujeong Mun, Ilkoo Ahn, and Siwoo Lee \\ Mibyeong Research Center, Korea Institute of Oriental Medicine, 1672 Yuseong-daero, Yuseong-gu, Daejeon 305-811, Republic of Korea
}

Correspondence should be addressed to Siwoo Lee; ifree72@gmail.com

Received 30 June 2017; Accepted 13 September 2017; Published 17 October 2017

Academic Editor: Kenji Watanabe

Copyright (C) 2017 Sujeong Mun et al. This is an open access article distributed under the Creative Commons Attribution License, which permits unrestricted use, distribution, and reproduction in any medium, provided the original work is properly cited.

Introduction. Facial diagnosis is a major component of the diagnostic method in traditional East Asian medicine. We investigated the association of quantitative facial color features with cold pattern using a fully automated facial color parameterization system. Methods. The facial color parameters of 64 participants were obtained from digital photographs using an automatic color correction and color parameter calculation system. Cold pattern severity was evaluated using a questionnaire. Results. The $a^{*}$ values of the whole face, lower cheek, and chin were negatively associated with cold pattern score (CPS) (whole face: $B=-1.048, P=0.021$; lower cheek: $B=-0.494, P=0.007$; chin: $B=-0.640, P=0.031$ ), while $b^{*}$ value of the lower cheek was positively associated with CPS $(B=0.234, P=0.019)$. The $a^{*}$ values of the whole face were significantly correlated with specific cold pattern symptoms including cold abdomen (partial $\rho=-0.354, P<0.01$ ) and cold sensation in the body (partial $\rho=-0.255, P<0.05$ ). Conclusions. $a^{*}$ values of the whole face were negatively associated with CPS, indicating that individuals with increased levels of cold pattern had paler faces. These findings suggest that objective facial diagnosis has utility for pattern identification.

\section{Introduction}

Pattern identification, also known as syndrome differentiation, is an essential component of the diagnostic process in traditional East Asian medicine (TEAM). Pattern identification is principally used to guide medical intervention; recent studies have suggested that identification improves the rate of successful treatment outcome for both traditional and conventional interventions $[1,2]$. Among the basic principles of pattern identification, cold pattern is believed to indicate the nature of imbalance in the body. Cold pattern is indicated when an individual has aversion to cold, preference for warmth, pale face, absence of thirst, cold limbs, clear urine in increased volume, and loose stools [3]. Recent studies indicate that cold pattern is common in women and individuals with low body mass index $[4,5]$. Cold pattern has been associated with alterations in metabolic rate [4], thyroid function $[6,7]$, the sympathetic nervous system $[5,8]$, the renin-angiotensin system [9], and glucose metabolism [5].

The evaluation of facial complexion has been used as a diagnostic method in TEAM and has also been studied outside of the context of TEAM [10]. Facial diagnosis has traditionally relied on subjective observations and is accordingly influenced by the practitioner's experience and personal knowledge. Thus, several studies have sought to develop an objective and reliable computer-assisted system for facial analysis and diagnosis, specifically in patients with hepatitis and diabetes mellitus [10-15]. Associations of quantified facial color with blood test parameters, health status, and TEAM patterns have also been widely investigated [14, 16-19].

Seo et al. [20] suggested that individuals with cold pattern tend to have pale or white facial color in the forehead, cheeks, and nose, as indicated by decreased $a^{*}$ components and increased $L^{*}$ components of the CIELAB color system, a universal standardized color system designed to approximate human vision [21]. Regions of interest in the study by Seo et al. were selected by three raters with good or excellent intrarater and interrater reliability; however, this region selection method may have been inconsistent across subjects based on the raters' subjective experiences and personal knowledge. In the present work, we investigated the association of quantitative facial color features with cold pattern using a computerized facial analysis system and color chart correction. We used a digital camera to acquire facial 


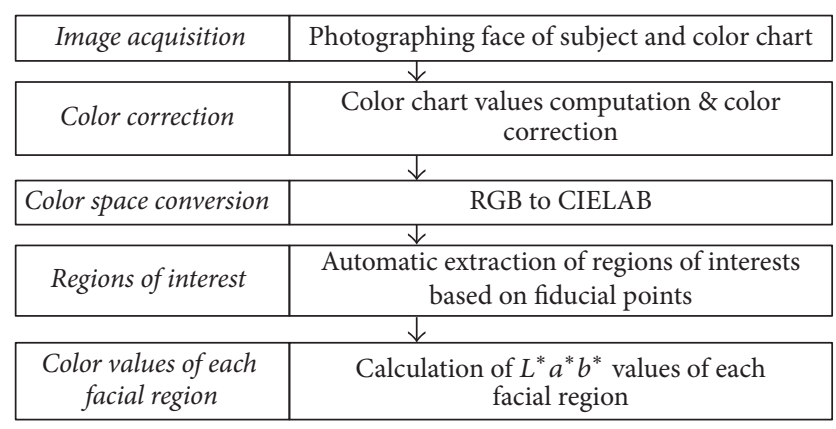

FIgURE 1: Schematic diagram of facial image analysis.

images with automated region selection and the extracted color features were transformed into the CIELAB color space. Automated region selection used both landmark detection and skin detection, which is more accurate than ROI determination by skin detection or approximate location information $[11,13]$. Then, associations of facial color parameters with cold pattern symptoms were investigated for each facial region. Automated region selection for regions including the forehead, upper/lower cheeks, nose, chin, and lips permits not only improved reproducibility and convenience, but also the ability to include whole representative facial color as a component of the analysis. Our study therefore represents a first attempt to create an objective method for facial diagnosis and pattern identification focusing on cold pattern.

\section{Methods}

2.1. Participants. This cross-sectional study was conducted between December 2014 and March 2015. Facial images and cold pattern data were derived from the Korean Medicine Data Center of the Korea Institute of Oriental Medicine [22]. Sixty-five individuals between the ages of 35 and 44 years, with a body mass index between 18.5 and $25 \mathrm{~kg} / \mathrm{m}^{2}$, and without any severe fatigue and pain, who are not currently receiving treatment for diseases were included in the study (see attachment for detailed inclusion and exclusion criteria in Supplementary Material available online at https://doi.org/10.1155/2017/9284856). The institutional review board of the hospital approved the study protocol (KHNMCIH 2014-09-010) and informed consent was obtained from all participants prior to inclusion in the study. Data from 64 participants were ultimately included in the analysis because one participant had missing data.

2.2. Facial Color. In this subsection, we describe the calculation method for $L^{*}, a^{*}$, and $b^{*}$ values of different facial regions. A schematic diagram of this process is shown in Figure 1.

2.2.1. Image Acquisition. Before facial image acquisition, the purpose and process were explained to participants. Participants were asked to wear a hairband so that their forehead and ears were exposed. Participants were seated comfortably in a chair and the camera was fixed with a tripod at a distance of $1.6 \mathrm{~m}$ from the subject. An x-rite color checker

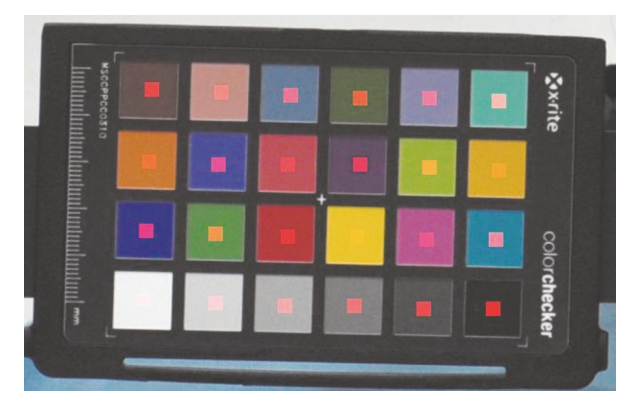

FIGURE 2: An example image of x-rite color checker passport used in color correction step. The reddish regions of each patch represent the center areas of each patch.

passport [23] was placed under each participant's chin as a reference color to correct exposure variability (see Figure 2). Frontal face images were acquired using a digital camera (Nikon D5100 with an 85 mm lens; Nikon Co., Ltd., Japan) equipped with a prime lens (a lens of fixed focal length) by a ruler. To reduce interrater bias, we prepared a strict standard operating procedure (SOP) for photo-taking and observed technicians periodically. If technicians were unable to produce uniform photographs in accordance with the SOP, they reacquired photographs until an appropriate level of accuracy was achieved. Facial images were acquired in the same location and with an outer fluorescent light source and saved at a resolution of $3696 \times 2448$ pixels in jpeg format.

2.2.2. Image Color Correction. Color correction requires reference color patches be placed in the given image, and then a conversion matrix is used to convert color chart values in the given image to original (target) color chart values. Multiplication of the given image and conversion matrix results in the corrected image. The Munsell color system [23] is commonly used as reference color patches to maintain color consistency across different illumination conditions. In this research, we used an $\mathrm{x}$-rite color checker passport (see Figure 2). The color checker consists of 24 color patches arranged in a $6 \times 4$ array, with colors selected to represent various natural objects. We used the method of Akkaynak et al. [25] to compute each patch's RGB color values in the color checker. This method automatically detects 24 patches and specifies the center of each patch (center areas are marked by red rectangles in Figure 2). The average RGB values of each patch's central region were calculated and 24 RGB values were obtained.

The following equation was established for the red channel to obtain the conversion matrix:

$$
\begin{aligned}
R_{1}^{\prime} & =\alpha_{0}+\alpha_{1} R_{1}+\alpha_{2} G_{1}+\alpha_{3} B_{1}+\alpha_{4} R_{1} G_{1}+\alpha_{5} G_{1} B_{1} \\
& +\alpha_{6} R_{1} B_{1}+\alpha_{7} R_{1}^{2}+\alpha_{8} G_{1}^{2}+\alpha_{9} B_{1}^{2} \\
R_{2}^{\prime} & =\alpha_{0}+\alpha_{1} R_{2}+\alpha_{2} G_{2}+\alpha_{3} B_{2}+\alpha_{4} R_{2} G_{2}+\alpha_{5} G_{2} B_{2} \\
& +\alpha_{6} R_{2} B_{2}+\alpha_{7} R_{2}^{2}+\alpha_{8} G_{2}^{2}+\alpha_{9} B_{2}^{2}
\end{aligned}
$$




$$
\begin{aligned}
& R_{24}^{\prime}=\alpha_{0}+\alpha_{1} R_{24}+\alpha_{2} G_{24}+\alpha_{3} B_{24}+\alpha_{4} R_{24} G_{24} \\
& \quad+\alpha_{5} G_{24} B_{24}+\alpha_{6} R_{24} B_{24}+\alpha_{7} R_{24}^{2}+\alpha_{8} G_{24}^{2}+\alpha_{9} B_{24}^{2},
\end{aligned}
$$

where $R_{1}^{\prime}, \ldots, R_{24}^{\prime}$ are the red channel values of the 1 st, $\ldots$, 24 th original (golden) patches, respectively, and $R_{1}, G_{1}$, and $B_{1}$ are the 1st red, green, and blue channel values of the 1st patch in a given image. Equation (1) can be expressed as matrix (2) as follows:

$$
\mathbf{R}^{\prime}=\mathbf{C A}_{R} \quad \text { where } \mathbf{R}^{\prime}=\left[\begin{array}{c}
R_{1}^{\prime} \\
\vdots \\
R_{24}^{\prime}
\end{array}\right], \mathbf{C}=\left[\begin{array}{cccccc}
1 & R_{1} & G_{1} & B_{1} & \cdots & B_{1}^{2} \\
1 & R_{2} & G_{2} & B_{2} & \cdots & B_{2}^{2} \\
& & & & \ddots & \\
1 & R_{24} & G_{24} & B_{24} & \cdots & B_{24}^{2}
\end{array}\right], \quad \mathbf{A}_{R}=\left[\begin{array}{c}
\alpha_{0} \\
\alpha_{1} \\
\vdots \\
\alpha_{9}
\end{array}\right]
$$

A least squares regression is used to solve for $\mathbf{A}_{R}$. The least squares solution of smallest norm of the linear system is given by

$$
\widehat{\mathbf{A}}_{R}=\mathbf{C}^{+} \mathbf{R}^{\prime},
$$

where $\mathbf{C}^{+}$is the pseudoinverse of $\mathbf{C}$. Now, we have a matrix $\widehat{\mathbf{A}}_{R}$ that converts red, green, and blue pixel values of a given image into a red pixel of a corrected image. This process is applied to other color channels $\left(\widehat{\mathbf{A}}_{G}\right.$ and $\left.\widehat{\mathbf{A}}_{B}\right)$ and least squares solutions of green and blue channels are obtained. Finally, the corrected image is computed by

$$
\left[\begin{array}{lll}
\widehat{\mathbf{R}} & \widehat{\mathbf{G}} & \widehat{\mathbf{B}}
\end{array}\right]=\mathbf{C}\left[\begin{array}{lll}
\widehat{\mathbf{A}}_{R} & \widehat{\mathbf{A}}_{G} & \widehat{\mathbf{A}}_{B}
\end{array}\right],
$$

where $\widehat{\mathbf{R}}, \widehat{\mathbf{G}}$, and $\widehat{\mathbf{B}}$ are red, green, and blue pixel values of a corrected image, respectively. The corrected image is then converted from the RGB color space to the CIELAB color space.

2.2.3. Facial Region of Interests (ROI) Extraction. To extract the same areas of facial ROIs for all participant face images, we used the facial landmark detector included in the Dlib library [26] which is an implementation of the method of Kazemi and Sullivan [27]. Figure 3 shows an example of the fiducial points on a frontal face (the original image is from Chicago Face Database [24] which is publicly released image database). The number next to the red dot indicates the order of the points in Figure 3. Using these points, we extracted facial ROIs as shown in Figure 4: forehead (1), upper cheek (2), nose (3), chin (4), lower cheek (5), lip (purple), and whole face region (red). The first five facial regions are extracted as a circle and are summarized in Table 1. Taking the nose region as an example, the center point position is determined as the center point of the 30th and 31st fiducial points. The radius of the nose region circle is 1.4 times the distance between the center point and the 31st point.

The lip region is determined by cubic spline data interpolated from points 49 to 60 for the outer boundary and from points 61 to 68 for the inner boundary as shown in Figure 5 . The whole face region is decided by spline interpolation from points 1 to 17 and points 69 to 73 . Here, the lip, nose tip, eyes, eyebrows, and nonskin regions are excluded from the whole face region. The nose tip region is extracted by spline interpolation from points 31 to 36 . The eyes and eyebrows regions are determined in a similar manner. Skin detection is performed by nonparametric histogram-based skin color modeling. We collected a database of skin and nonskin patches from different images from a variety of people under different illumination conditions. Two three-dimensional histogram color models (one for skin and another for nonskin) are constructed in the 24-bit RGB color space. Both histograms (size, 32 bins per color channel) have 32,768 bins each. Each bin of the skin model histogram stores an integer counting the number of times that color value occurs in the entire database of skin images and the same approach is applied to the nonskin model histogram. The counts of each cube histogram are converted into a discrete probability distribution. Finally, an unknown RGB pixel $\mathbf{x}$ is labeled as skin or nonskin using the Bayesian classifier as follows:

$$
\mathbf{x}= \begin{cases}\text { skin, } & \text { if } p(\mathbf{x} \mid \text { skin })>p(\mathbf{x} \mid \text { nonskin }) \\ \text { nonskin, } & \text { otherwise. }\end{cases}
$$

After obtaining facial ROIs, each region's trimmed averages of CIELAB $L^{*}, a^{*}$, and $b^{*}$ values are computed, where the trimmed average is the mean after discarding the upper and lower quartiles of the distributions. The trimmed mean is a suitable estimator for this study because it excludes the influence of outlying colors in very confined areas such as in rashes, wrinkles, dots, scars, and whiskers in each ROI, instead of reflecting the overall color of the region. The proposed system was implemented in Matlab R2016a (Matlab and Statistics and Machine Learning Toolbox, The MathWorks, Inc., Natick, Massachusetts, United States) in a Win64 environment.

2.3. Cold Pattern. The cold pattern questionnaire consisted of eight items that addressed cold pattern symptoms [28], including aversion to cold, preference for heat, cold abdomen, cold hands/feet, cold sensation in the body, pale face, drinking warm water, and clear urine. The questionnaire was selfadministered and each question was evaluated on a 4-point scale: $1=$ strongly disagree; $2=$ disagree; $3=$ agree; and $4=$ strongly agree. The cold pattern score (CPS) was calculated as the sum of all eight items and ranged from 0 to 32, with 
TABLE 1: The extraction method of facial regions of interests.

\begin{tabular}{|c|c|c|}
\hline \multirow{2}{*}{ Region (region number) } & \multicolumn{2}{|c|}{ Description } \\
\hline & Center point position & Radius \\
\hline Whole face & \multicolumn{2}{|c|}{$\begin{array}{c}\text { Determined by spline interpolation from points } 1 \text { to } 17 \text { and from } 69 \text { to } 73 \text {. Lip, nose } \\
\text { tip (31 36), eyes }(43 \sim 48,37 \sim 40) \text {, eyebrows }(23 \sim 27 \& 77 \sim 79,18 \sim 22 \& \text { \&4 76) } \\
\text { regions and nonskin regions are excluded }\end{array}$} \\
\hline Forehead (1) & $(\mathrm{pt} 20+\mathrm{pt} 25) / 2+(\mathrm{pt} 28-\mathrm{pt} 63) * 0.2$ & Distance (center point, pt 22$) * 0.7$ \\
\hline Upper cheek (2) & $\begin{array}{l}\text { Left: pt31 } * 0.4+(\mathrm{pt} 15+\mathrm{pt} 16) * 0.3 \\
\text { Right: pt31 } * 0.4+(\mathrm{pt} 2+\mathrm{pt} 3) * 0.3\end{array}$ & $\begin{array}{l}\text { Distance (center point, pt47) } * 0.8 \\
\text { Distance (center point, pt } 42) * 0.8\end{array}$ \\
\hline Lower cheek (5) & $\begin{array}{l}\text { Left: pt } 31 * 0.25+\text { pt } 13 * 0.75 \\
\text { Right: pt } 31 * 0.25+\text { pt } 5 * 0.75\end{array}$ & $\begin{array}{l}\text { Distance (center point, pt13) } * 0.7 \\
\text { Distance (center point, pt5) } * 0.7\end{array}$ \\
\hline Nose (3) & $(\mathrm{pt} 30+\mathrm{pt} 31) / 2$ & Distance (center point, pt31) $* 1.4$ \\
\hline Chin (4) & pt9 $* 0.4+$ pt58 $* 0.6$ & Distance (center point, pt58) $* 0.8$ \\
\hline Lip & $\begin{array}{l}\text { Determined by spline interpolation fro } \\
\text { to } 68\end{array}$ & 60 (outer) and from 61 \\
\hline
\end{tabular}

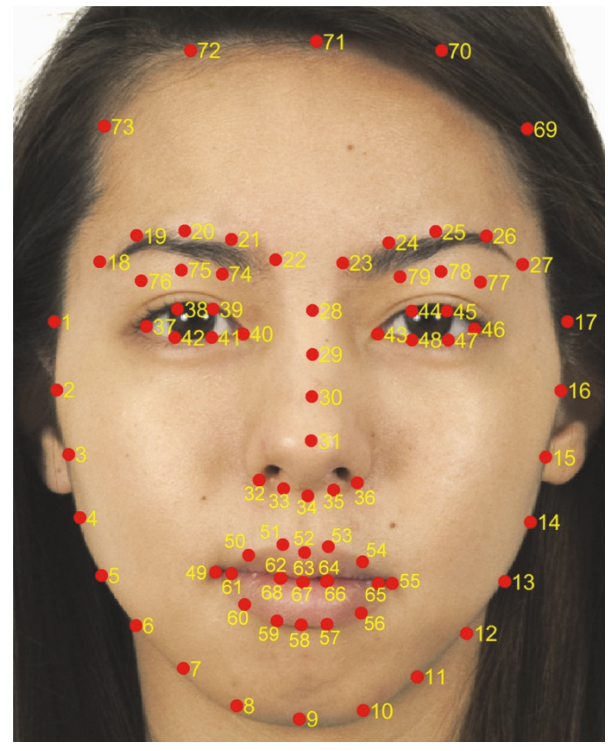

Figure 3: An example of the fiducial points on a frontal face. The original image is from Chicago Face Database [24] which is publicly released image database.

higher scores indicating more advanced states of cold pattern. Cronbach's coefficient for this tool was previously reported to be 0.79 [29].

2.4. Statistical Analysis. The characteristics of participants are presented as the mean and standard deviation and categorical data are presented as the percentage. To determine the adjusted association of facial colors and cold pattern symptoms, a linear regression analysis was conducted with CPS as a dependent variable and facial color parameters as independent variables, adjusted for sex and age. To assess the association of facial colors and specific cold pattern symptoms, partial correlation coefficients were computed adjusting for sex and age. A $P$ value of less than 0.05 was considered to be statistically significant. Statistical analyses were performed using SPSS 22.0 (IBM, Chicago, IL, USA).

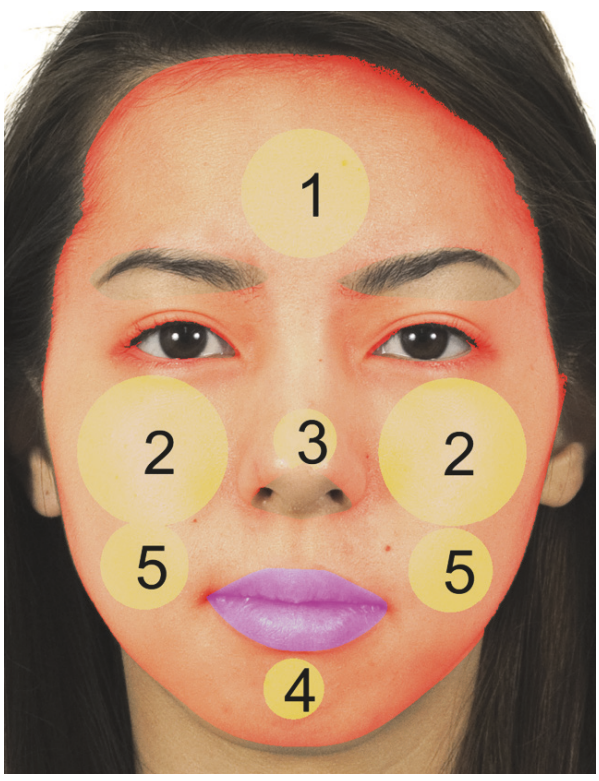

FIGURE 4: Facial regions of interests (ROI). ROIs (yellow circles) and lip (purple) and whole area of face (reddish). 1: forehead, 2: upper cheek, 3: nose, 4: chin, and 5: lower cheek. The original image is from [24].

\section{Results}

3.1. Characteristics of Participants. The subjects were 32 male participants and 32 female participants with a mean age of $39.2 \pm 3.2$ years and mean cold pattern score of $20.0 \pm 3.6$ as shown in Table 2 . The mean values of $L^{*}, a^{*}$, and $b^{*}$ for each facial region are presented in Table 2. $L^{*}$ values were highest in the nose region, $a^{*}$ values were highest in the lip region, and $b^{*}$ values were highest in the forehead region.

3.2. Association of Facial Color with CPS. The results of a multiple regression analysis showed that $a^{*}$ value of the whole face was negatively associated with CPS $(B=-1.048, P=$ $0.021)$. In a regional analysis, $a^{*}$ values of the lower cheek 
TABLE 2: Characteristics of participants.

\begin{tabular}{|c|c|c|c|c|}
\hline Number of participants & 64 & $\begin{array}{c}\text { Male } \\
32(50.0 \%) \\
\end{array}$ & $\begin{array}{c}\text { Female } \\
32(50.0 \%)\end{array}$ & $P$ value \\
\hline Age (yr) & $39.2 \pm 3.2$ & $38.8 \pm 3.2$ & $39.7 \pm 3.2$ & 0.2588 \\
\hline CPS & $20.0 \pm 3.6$ & $18.9 \pm 3.3$ & $21.1 \pm 3.5$ & 0.0126 \\
\hline \multicolumn{5}{|l|}{$L^{*}$ (a.u.) } \\
\hline Whole face & $65.5 \pm 3.2$ & $64.4 \pm 3.8$ & $66.5 \pm 2.0$ & 0.0085 \\
\hline Forehead & $73.3 \pm 3.8$ & $74.1 \pm 4.3$ & $72.5 \pm 3.3$ & 0.1053 \\
\hline Upper cheek & $66.0 \pm 3.5$ & $129.1 \pm 7.6$ & $135.1 \pm 4.7$ & 0.0004 \\
\hline Lower cheek & $60.7 \pm 3.3$ & $119.7 \pm 7.8$ & $123.0 \pm 5.0$ & 0.0515 \\
\hline Nose & $78.0 \pm 3.8$ & $77.5 \pm 4.3$ & $78.6 \pm 3.3$ & 0.2404 \\
\hline Chin & $57.5 \pm 5.2$ & $55.2 \pm 4.9$ & $59.9 \pm 4.5$ & 0.0002 \\
\hline Lip & $46.2 \pm 3.7$ & $45.6 \pm 4.7$ & $46.7 \pm 2.5$ & 0.2686 \\
\hline \multicolumn{5}{|l|}{$a^{*}$ (a.u.) } \\
\hline Whole face & $13.0 \pm 1.5$ & $13.5 \pm 1.6$ & $12.4 \pm 1.2$ & 0.0018 \\
\hline Forehead & $11.3 \pm 1.9$ & $11.6 \pm 2.1$ & $11.1 \pm 1.6$ & 0.3374 \\
\hline Upper cheek & $14.8 \pm 1.7$ & $31.1 \pm 3.5$ & $28.3 \pm 2.8$ & 0.0008 \\
\hline Lower cheek & $15.0 \pm 1.7$ & $30.4 \pm 3.5$ & $29.4 \pm 3.0$ & 0.2158 \\
\hline Nose & $11.8 \pm 2.0$ & $12.4 \pm 2.2$ & $11.2 \pm 1.6$ & 0.0134 \\
\hline Chin & $16.9 \pm 2.1$ & $17.0 \pm 2.1$ & $16.9 \pm 2.2$ & 0.9203 \\
\hline Lip & $24.2 \pm 2.4$ & $23.7 \pm 2.1$ & $24.6 \pm 2.6$ & 0.1331 \\
\hline \multicolumn{5}{|l|}{$b^{*}$ (a.u.) } \\
\hline Whole face & $22.2 \pm 2.2$ & $21.3 \pm 2.2$ & $23.1 \pm 1.8$ & 0.0009 \\
\hline Forehead & $24.2 \pm 2.8$ & $23.3 \pm 2.7$ & $25.2 \pm 2.6$ & 0.0041 \\
\hline Upper cheek & $22.8 \pm 2.3$ & $44.3 \pm 4.8$ & $46.9 \pm 4.2$ & 0.0242 \\
\hline Lower cheek & $23.0 \pm 2.4$ & $45.0 \pm 5.2$ & $47.0 \pm 4.3$ & 0.0970 \\
\hline Nose & $20.5 \pm 2.9$ & $19.6 \pm 2.6$ & $21.4 \pm 3.0$ & 0.0131 \\
\hline Chin & $22.2 \pm 3.0$ & $20.9 \pm 2.8$ & $23.4 \pm 2.6$ & 0.0004 \\
\hline Lip & $12.3 \pm 1.8$ & $11.9 \pm 2.1$ & $12.7 \pm 1.3$ & 0.0831 \\
\hline
\end{tabular}

CPS, cold pattern score; $L^{*}$, light $(+)$ to dark $(-) ; a^{*}$, red $(+)$ to green $(-) ; b^{*}$, yellow $(+)$ to blue $(-)$. Statistically significant differences between group means as determined by one-way ANOVA are in bold.

TABLE 3: Multiple regression analysis for the association of facial color parameters and cold pattern score.

\begin{tabular}{|c|c|c|c|c|c|c|c|c|}
\hline \multirow{2}{*}{ Region } & \multicolumn{2}{|c|}{$L^{*}$} & \multicolumn{2}{|c|}{$a^{*}$} & \multicolumn{2}{|c|}{$b^{*}$} & \multirow{2}{*}{$R^{2}$} & \multirow{2}{*}{ Adjusted $R^{2}$} \\
\hline & $B$ & $P$ value & $B$ & $P$ value & $B$ & $P$ value & & \\
\hline Whole & -0.156 & 0.463 & -1.048 & 0.021 & 0.314 & 0.173 & 0.244 & 0.178 \\
\hline Forehead & 0.076 & 0.667 & -0.448 & 0.215 & 0.205 & 0.212 & 0.222 & 0.153 \\
\hline Upper cheek & -0.029 & 0.766 & -0.370 & 0.052 & 0.147 & 0.153 & 0.229 & 0.162 \\
\hline Lower cheek & -0.120 & 0.213 & -0.494 & 0.007 & 0.234 & 0.019 & 0.270 & 0.206 \\
\hline Nose & -0.007 & 0.967 & -0.264 & 0.487 & 0.201 & 0.256 & 0.151 & 0.076 \\
\hline Chin & -0.126 & 0.374 & -0.640 & 0.031 & 0.231 & 0.191 & 0.205 & 0.135 \\
\hline Lip & -0.093 & 0.527 & -0.214 & 0.262 & 0.424 & 0.171 & 0.142 & 0.067 \\
\hline
\end{tabular}

Multiple regression analysis was used adjusting for sex and age. Statistically significant results are in bold; $L^{*}$, light $(+)$ to dark (-); $a^{*}$, red $(+)$ to green $(-) ; b^{*}$, yellow (+) to blue (-); $B$, unstandardized coefficients.

and chin regions were negatively associated with CPS (lower cheek: $B=-0.494, P=0.007$; chin: $B=-0.640, P=0.031$, while $b^{*}$ value of the lower cheek was positively associated with CPS ( $B=0.234, P=0.019)$ (Table 3$)$.

3.3. Correlation of Facial Color with Specific Cold Pattern Symptoms. $L^{*}$ values of the forehead and chin were significantly correlated with cold hands/feet (forehead: partial correlation coefficient $=0.284, P<0.05)$ and cold sensation in the body (forehead: partial correlation coefficient $=0.292$, $P<0.05$; chin: partial correlation coefficient $=0.258, P<$ $0.05) \cdot a^{*}$ values of the whole face were significantly correlated with cold abdomen (partial correlation coefficient $=-0.354$, $P<0.01$ ), cold sensation in the body (partial correlation coefficient $=-0.255, P<0.05$ ), and pale face (partial correlation coefficient $=-0.349, P<0.01) . a^{*}$ values of 
TABLE 4: Partial correlation coefficients between facial color and cold pattern questions.

\begin{tabular}{|c|c|c|c|c|c|c|c|c|}
\hline & $\begin{array}{l}\text { Aversion to } \\
\text { cold }\end{array}$ & $\begin{array}{l}\text { Preference } \\
\text { for heat }\end{array}$ & $\begin{array}{c}\text { Cold } \\
\text { abdomen }\end{array}$ & $\begin{array}{c}\text { Cold } \\
\text { hands/feet }\end{array}$ & $\begin{array}{c}\text { Cold } \\
\text { sensation in } \\
\text { body }\end{array}$ & Pale face & $\begin{array}{c}\text { Drink warm } \\
\text { water }\end{array}$ & Clear urine \\
\hline \multicolumn{9}{|l|}{$L^{*}$} \\
\hline Whole face & 0.018 & 0.162 & 0.119 & 0.147 & 0.214 & 0.215 & 0.121 & 0.095 \\
\hline Forehead & 0.053 & 0.148 & 0.194 & $0.284^{*}$ & $0.292^{*}$ & 0.089 & 0.099 & 0.102 \\
\hline Upper cheek & 0.045 & 0.188 & 0.103 & 0.118 & 0.231 & 0.166 & 0.176 & 0.082 \\
\hline Lower cheek & 0.016 & 0.174 & 0.137 & 0.100 & 0.162 & 0.223 & 0.026 & 0.127 \\
\hline Nose & 0.047 & 0.033 & -0.018 & 0.076 & 0.182 & 0.102 & 0.035 & 0.108 \\
\hline Chin & 0.076 & 0.138 & 0.070 & 0.088 & $0.258^{*}$ & 0.184 & 0.007 & -0.016 \\
\hline Lip & -0.039 & 0.159 & -0.105 & -0.129 & 0.071 & 0.046 & 0.099 & 0.178 \\
\hline \multicolumn{9}{|l|}{$a^{*}$} \\
\hline Whole face & -0.188 & -0.196 & $-0.354^{* *}$ & -0.184 & $-0.255^{*}$ & $-0.349^{* *}$ & -0.048 & -0.029 \\
\hline Forehead & -0.164 & -0.148 & $-0.394^{* *}$ & -0.216 & -0.218 & -0.185 & -0.012 & -0.105 \\
\hline Upper cheek & -0.212 & $-0.263^{*}$ & $-0.291^{*}$ & -0.135 & -0.243 & $-0.289^{*}$ & -0.109 & 0.042 \\
\hline Lower cheek & -0.175 & -0.188 & $-0.296^{*}$ & -0.151 & -0.209 & $-0.395^{* *}$ & 0.003 & -0.064 \\
\hline Nose & -0.087 & -0.027 & -0.132 & -0.134 & -0.108 & -0.21 & -0.022 & -0.134 \\
\hline Chin & -0.150 & -0.138 & -0.241 & -0.107 & $-0.296^{*}$ & $-0.411^{* *}$ & 0.007 & -0.002 \\
\hline Lip & -0.144 & -0.082 & -0.006 & 0.128 & -0.021 & -0.243 & $-0.333^{* *}$ & 0.073 \\
\hline \multicolumn{9}{|l|}{$b^{*}$} \\
\hline Whole face & 0.176 & 0.153 & 0.065 & 0.049 & 0.107 & 0.113 & 0.125 & 0.241 \\
\hline Forehead & 0.169 & 0.158 & 0.052 & 0.032 & 0.098 & 0.112 & 0.102 & 0.223 \\
\hline Upper cheek & 0.159 & 0.143 & 0.019 & 0.105 & 0.160 & 0.146 & 0.089 & 0.236 \\
\hline Lower cheek & $0.268^{*}$ & 0.191 & 0.076 & 0.100 & 0.115 & 0.067 & 0.112 & 0.216 \\
\hline Nose & 0.095 & 0.057 & 0.060 & 0.121 & 0.093 & 0.175 & 0.111 & 0.208 \\
\hline Chin & 0.227 & 0.081 & 0.123 & -0.010 & 0.034 & 0.046 & 0.108 & 0.144 \\
\hline Lip & 0.093 & 0.233 & 0.096 & -0.037 & 0.067 & 0.037 & 0.111 & 0.184 \\
\hline
\end{tabular}

Partial correlation coefficients adjusted for sex and age. Statistically significant results are in bold; $L^{*}$, light $(+)$ to dark $(-) ; a^{*}$, red $(+)$ to green $(-) ; b^{*}$, yellow (+) to blue $(-) ;{ }^{*} P<0.05 ;{ }^{* *} P<0.01$.

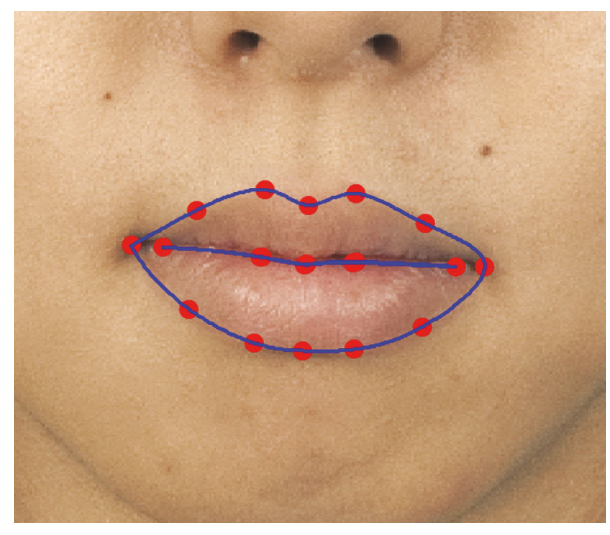

FIgURE 5: An example of the lip region extraction by spline interpolation. The boundaries of lip region and fiducial points are marked with blue line and red dots, respectively. The original image is from [24].

other regions including the forehead, upper and lower cheek, chin, and lip were significantly correlated with preference for heat, cold abdomen, cold sensation in the body, pale face, and drinking warm water. $b^{*}$ value was correlated with aversion to cold only in the lower cheek (partial correlation coefficient $=0.268, P<0.05$ ) (Table 4).

\section{Discussion}

The present study conducted a quantitative assessment of the relationship between facial color features and cold pattern and found that $a^{*}$ values of the whole face, lower cheek, and chin were negatively associated with CPS. Moreover, $a^{*}$ values of the whole face, forehead, cheek, and chin were negatively associated with cold pattern symptoms including cold abdomen and cold sensation in the body. Among CIELAB color components, $a^{*}$ was the most relevant to cold pattern in our cohort and $a^{*}$ denotes color on a scale from green (negative values) to red (positive values), such that a negative association between $a^{*}$ and CPS is consistent with the TEAM theory that individuals with cold pattern have pale faces [3]. $a^{*}$ values are primarily affected by hemoglobin concentration, blood perfusion to the skin, and blood oxygenation $[30,31]$. Although the biological basis of cold pattern remains unclear, cold pattern is often presumed to be related to decreased metabolic rate or diminished function of the whole 
body [32-34]. It is plausible that a metabolically inactive state demands decreased oxygen in tissue, resulting in decreased skin perfusion and oxygenation and thus decreased $a^{*}$ values in the skin. This hypothesis is consistent with a previous study that reported an association between skin $a^{*}$ value and physical fitness following repeated bouts of dynamic exercise [35]; in this study, increased $a^{*}$ values were assumed to be an adaptive response to demand for increased blood flow to cardiac and skeletal muscle tissues.

We also identified a positive association between $b^{*}$ values in the lower cheek area and CPS. $b^{*}$ values are mainly affected by melanin and carotenoids [31]; however, it is also reported that $b^{*}$ values in skin are negatively associated with hemoglobin concentration [36]. Thus, a relationship of high CPS with decreased $a^{*}$ values and increased $b^{*}$ values could be explained by decreased cutaneous blood flow or hemoglobin concentrations in individuals with cold pattern, although the specific mechanism of this effect needs to be clarified in future studies.

In our cohort, the colors of the lower cheek and whole face explained the variance of CPS better than the colors of other regions. This is consistent with a previous finding that the colors of the cheek were more indicative of TEAM patterns than other regions (notably, color of the whole face was not included in the analysis) [20]. The cheek is a facial region with high cutaneous blood flow [37]. Blood vessels in the cheek are wider in diameter and located closer to the skin surface than blood vessels in other regions of the face [38]. These traits contribute to the easy visibility of blood perfusion and oxygenation in the cheek. Yet, the reason for less of an association between color of the upper cheek and cold pattern is unclear. The upper cheek is more exposed to sunlight than the lower cheek, such that the influence of sun exposure and increased melanin or freckling may have interfered with the results. The reflection of light on the upper cheek when photos were taken may have also affected the results given the use of a fluorescent light on the ceiling as a light source.

Cold pattern is traditionally diagnosed with comprehensive patient screening and the presence of multiple symptoms rather than a single symptom. In this study, correlations of color parameters with specific cold pattern symptoms were analyzed to verify that the color parameters related to multiple component symptoms of cold pattern and not just the item related to facial color (i.e., self-determined level of paleness of the face). The results showed that $a^{*}$ of the whole face had a weak but significant negative correlation with the severity of cold sensation in the abdomen and cold sensation in the body. Interestingly but not surprisingly, correlations were weak between self-determined level of paleness of the face and color parameters, emphasizing the importance of objectivity in facial diagnosis.

The development of an objective pattern identification process has long been desired in order to standardize the diagnostic process of TEAM. Recent studies have shown that various objective variables have significant associations with cold pattern including anthropometric measures such as body mass index [39], resting metabolic rate [4], heart rate variability parameters [8], adiponectin [40], norepinephrine, and changes in glucose and insulin during oral glucose tolerance testing [5]. Together with these previous findings, we hope that our results contribute to the formation of an objective pattern identification system and understanding the underlying mechanism of cold pattern.

Several study limitations should be considered when interpreting our findings. First, we used a subjective selfreport cold pattern questionnaire consisting of representative symptoms of cold pattern that were perceived by participants, whereas the traditional diagnosis of a pattern includes a comprehensive inspection (e.g., listening, smelling), inquiry, and palpation of the patient by practitioner (i.e., "the four diagnostic methods"). We selected this questionnaire instead of practitioner-based diagnosis because of the widespread use of structured questionnaires for assessing TEAM patterns in research and the established validity and reliability of the questionnaire used in our study [20,41,42]. Additionally, the four diagnostic methods used by a practitioner might lead to different diagnoses for the same individual, resulting in considerable variability across practitioners $[43,44]$. Second, although we considered sex and age as covariates, several external factors potentially affecting skin pigmentation were not considered in our analysis. Futures studies should consider the level of sun exposure, which is the most significant external factor affecting skin pigmentation. Third, this study was conducted at a single center with a small sample size. To improve the generalizability of our results, an analysis should be conducted with a larger sample and more strict control of the experimental environment (e.g., light source during photography). Fourth, gloss and luster of the face were not analyzed in this study. In addition to coloring, gloss and luster are important features for facial complexion diagnosis. Thus, future work should assess relationships between facial gloss parameters and specific cold pattern symptoms. Finally, since the results of this study indicate significant associations between color parameters and cold pattern, it will be useful to create a classification model using machine learning techniques for cold pattern identification in the future.

\section{Conclusions}

We investigated the relationship between facial color features and cold pattern using a digital camera and a fully automated facial color parameterization system. $a^{*}$ values of the whole face, lower cheek, and chin were negatively associated with cold pattern severity. These results suggest that facial diagnosis can be performed objectively for pattern identification.

\section{Conflicts of Interest}

The authors declare that there are no conflicts of interest regarding the publication of this paper.

\section{Authors' Contributions}

Sujeong Mun analyzed the data and drafted the manuscript. Ilkoo Ahn proposed automatic color correction and color parameter calculation system and drafted the manuscript on the system. Siwoo Lee conceived of the study and participated in its design and coordination. All of the authors read and 
approved the final manuscript. Sujeong Mun and Ilkoo Ahn are equal contributors.

\section{Acknowledgments}

This work was supported by the Ministry of Science, ICT \& Future Planning [NRF-2014M3A9D7034351] and the Korea Institute of Oriental Medicine [K17092].

\section{References}

[1] A. S. Ferreira and A. J. Lopes, "Chinese medicine pattern differentiation and its implications for clinical practice," Chinese Journal of Integrative Medicine, vol. 17, no. 11, pp. 818-823, 2011.

[2] C. Lu, Q.-I. Zha, A.-I. Chang, Y. T. He, and A.-P. Lu, "Pattern differentiation in traditional chinese medicine can help define specific indications for biomedical therapy in the treatment of rheumatoid arthritis," The Journal of Alternative and Complementary Medicine, vol. 15, no. 9, pp. 1021-1025, 2009.

[3] Z. Bing and H. Wang, Diagnostics of Traditional Chinese Medicine, Singing Dragon, London, UK, 2010.

[4] S. Mun, S. Kim, K.-H. Bae, and S. Lee, "Cold and Spleen-Qi Deficiency Patterns in Korean Medicine Are Associated with Low Resting Metabolic Rate," Evidence-Based Complementary and Alternative Medicine, vol. 2017, Article ID 9532073, 2017.

[5] D. D. Pham, J. Lee, G. Kim, J. Song, J. Kim, and C. H. Leem, "Relationship of the Cold-Heat Sensation of the Limbs and Abdomen with Physiological Biomarkers," Evidence-Based Complementary and Alternative Medicine, vol. 2016, Article ID 2718051, 2016.

[6] K. Nagashima, T. Yoda, T. Yagishita, A. Taniguchi, T. Hosono, and K. Kanosue, "Thermal regulation and comfort during a mild-cold exposure in young Japanese women complaining of unusual coldness," Journal of Applied Physiology, vol. 92, no. 3, pp. 1029-1035, 2002.

[7] M. Lee and Y. Choi, "Systemic reviews of domestic experimental studies of herbal medicines used for hypothyroidism since 2000," The Journal of Internal Korean Medicine, vol. 36, no. 4, pp. 570-581, 2015.

[8] Y.-J. Park, J.-M. Lee, and Y.-B. Park, "Relationships between oriental medical pattern diagnosis and cardiovascular autonomic function," European Journal of Integrative Medicine, vol. 5, no. 6, pp. 506-513, 2013.

[9] T. Ma, C. Tan, H. Zhang, M. Wang, W. Ding, and S. Li, "Bridging the gap between traditional Chinese medicine and systems biology: The connection of Cold Syndrome and NEI network," Molecular BioSystems, vol. 6, no. 4, pp. 613-619, 2010.

[10] X. Li, F. Li, Y. Wang, P. Qian, and X. Zheng, "Computer-aided disease diagnosis system in TCM based on facial image analysis," International Journal of Functional Informatics and Personalised Medicine, vol. 2, no. 3, pp. 303-314, 2009.

[11] X. Wang, B. Zhang, Z. Guo, and D. Zhang, "Facial image medical analysis system using quantitative chromatic feature," Expert Systems with Applications, vol. 40, no. 9, pp. 3738-3746, 2013.

[12] M. Liu and Z. Guo, "Hepatitis diagnosis using facial color image," in Medical Biometrics, vol. 4901 of Lecture Notes in Computer Science, pp. 160-167, Springer, Berlin, Germany, 2007.

[13] C. Zhao, G. Li, F. Li, Z. Wang, and C. Liu, "Qualitative and quantitative analysis for facial complexion in traditional chinese medicine," BioMed Research International, vol. 2014, Article ID 207589, 17 pages, 2014.
[14] C. Yanya, L. Fufeng, and W. Yiqin, "Analysis on Facial Color Diagnosis for CHD Patients," Chinese Arch. Tradit. Chinese Med, vol. 31, no. 9, pp. 1-2, 2013.

[15] B. Zhang, B. V. K. V. Kumar, and D. Zhang, "Noninvasive diabetes mellitus detection using facial block color with a sparse representation classifier," IEEE Transactions on Biomedical Engineering, vol. 61, no. 4, pp. 1027-1033, 2014.

[16] J. Xu, H. Wu, L. Lu et al., "Analysis of different health status based on characteristics of the facial spectrum photometric color," Sheng Wu Yi Xue Gong Cheng Xue Za Zhi, vol. 29, no. 6, pp. 1062-1067, 2012 (Chinese).

[17] H.-J. Wu, J.-T. Xu, L.-M. Lu, X. Chen, L.-P. Tu, and Z.-F. Zhang, "Facial spectrum and color characteristics of five viscera reflecting on the face in sub-health status," Journal of Chinese Integrative Medicine, vol. 10, no. 1, pp. 59-66, 2012.

[18] L. Fu-feng, Z. Xiao-yan, and Y. Wang, "Studies on the Relationship Between the Facial Complexion Characters and Kidney Function in TCM Clinical Syndrome of Chronic Renal failure," Chinese Arch. Tradit. Chinese Med, vol. 29, no. 9, pp. 7-10, 2011.

[19] B. C. Jones, A. C. Hahn, C. I. Fisher et al., "Facial coloration tracks changes in women's estradiol," Psychoneuroendocrinology, vol. 56, pp. 29-34, 2015.

[20] J.-H. Seo, Y.-B. Park, and Y.-J. Park, "Reliable facial color analysis using a digital camera and its relationship with pathological patterns: A pilot study," European Journal of Integrative Medicine, vol. 6, no. 3, pp. 322-327, 2014.

[21] M. Edwards, A. Gozdzik, K. Ross, J. Miles, and E. J. Parra, "Technical note: Quantitative measures of iris color using high resolution photographs," American Journal of Physical Anthropology, vol. 147, no. 1, pp. 141-149, 2012.

[22] H. J. Jin, Y. Baek, H. S. Kim, J. Ryu, and S. Lee, "Constitutional multicenter bank linked to Sasang constitutional phenotypic data," BMC Complementary and Alternative Medicine, vol. 15, article 46, 2015.

[23] R. D. Myers, “ColorChecker Passport Technical Review," Tech. Rep., 2010.

[24] D. S. Ma, J. Correll, and B. Wittenbrink, "The Chicago face database: A free stimulus set of faces and norming data," Behavior Research Methods, vol. 47, no. 4, pp. 1122-1135, 2015.

[25] D. Akkaynak, T. Treibitz, B. Xiao et al., "Use of commercial off-the-shelf digital cameras for scientific data acquisition and scene-specific color calibration," Journal of the Optical Society of America A: Optics and Image Science, and Vision, vol. 31, no. 2, pp. 312-321, 2014.

[26] D. E. King, "Dlib-ml: a machine learning toolkit," J. Mach. Learn. Res, vol. 10, pp. 1755-1758, 2009.

[27] V. Kazemi and J. Sullivan, "One millisecond face alignment with an ensemble of regression trees," in Proceedings of the IEEE Conference on Computer Vision and Pattern Recognition (CVPR '14), pp. 1867-1874, Columbus, Ohio, USA, June 2014.

[28] K. H. Bae, Y. Yoon, M. Yeo et al., "Development on the Questionnaire of Cold-Heat Pattern Identification Based on Usual Symptoms for Health Promotion," J. Physiol. Pathol. Korean Med, vol. 30, no. 2, pp. 116-123, 2016.

[29] M. Yeo, K. Park, K. Bae, E. Jang, and Y. Lee, "Development on the Questionnaire of Cold-Heat Pattern Identification Based on Usual Symptoms for Health Promotion - Focused on Reliability Study," Journal of Physiology \& Pathology in Korean Medicine, vol. 30, no. 2, p. 116, 2016.

[30] H. Takiwaki, "Measurement of skin color : Practical application and theoretical considerations," Journal of Medical Investigation, vol. 44, no. 3-4, pp. 121-126, 1998. 
[31] I. D. Stephen, M. J. Law Smith, M. R. Stirrat, and D. I. Perrett, "Facial skin coloration affects perceived health of human faces," International Journal of Primatology, vol. 30, no. 6, pp. 845-857, 2009.

[32] T. Deng, Practical Diagnosis in Traditional Chinese Medicine, Churchill Livingstone, Edinburgh, Scotland, 1999.

[33] T. Yoshino, K. Katayama, K. Munakata et al., "Statistical analysis of Hie (cold sensation) and Hiesho (cold disorder) in Kampo Clinic," Evidence-Based Complementary and Alternative Medicine, vol. 2013, Article ID 398458, 8 pages, 2013.

[34] Z. Xu, Q. Chen, and Q. Sun, "Heat syndrome of traditional Chinese medicine," Chinese Journal of Comparative Medicine, vol. 19, no. 3, pp. 53-56, 2009.

[35] J. M. Johnson, "Physical training and the control of skin blood flow, Medicine \& Science in Sports \& Exercise, vol. 30, no. 3, pp. 382-386, 1998.

[36] G. N. Stamatas, B. Z. Zmudzka, N. Kollias, and J. Z. Beer, "Noninvasive measurements of skin pigmentation in situ," Pigment Cell Research, vol. 17, no. 6, pp. 618-626, 2004.

[37] C. V. Wa and H. I. Maibach, "Mapping the human face: Biophysical properties," Skin Research and Technology, vol. 16, no. 1, pp. 38-54, 2010.

[38] J. K. Wilkin, "Why is flushing limited to a mostly facial cutaneous distribution?" Journal of the American Academy of Dermatology, vol. 19, no. 2 I, pp. 309-313, 1988.

[39] B. J. Lee, J. C. Lee, J. Nam, and J. Y. Kim, "Prediction of cold and heat patterns using anthropometric measures based on machine learning," Chinese Journal of Integrative Medicine, pp. 1-8, 2016.

[40] A. Y. Park and S. Cha, "Effects of cold sensitivity in the extremities on circulating adiponectin levels and metabolic syndrome in women," BMC Complementary and Alternative Medicine, vol. 17, no. 1, article no. 150, 2017.

[41] B.-K. Kang, T.-Y. Park, J. A. Lee et al., "Reliability and validity of the Korean standard pattern identification for stroke (K-SPIStroke) questionnaire," BMC Complementary and Alternative Medicine, vol. 12, article 55, 2012.

[42] C. A. Berle, D. Cobbin, N. Smith, and C. Zaslawski, "A novel approach to evaluate traditional Chinese medicine treatment outcomes using pattern identification," The Journal of Alternative and Complementary Medicine, vol. 16, no. 4, pp. 357-367, 2010.

[43] C. J. Hogeboom, K. J. Sherman, and D. C. Cherkin, "Variation in diagnosis and treatment of chronic low back pain by traditional Chinese medicine acupuncturists," Complementary Therapies in Medicine, vol. 9, no. 3, pp. 154-166, 2001.

[44] B. Zell, J. Hirata, A. Marcus, B. Ettinger, A. Pressman, and K. M. Ettinger, "Diagnosis of symptomatic postmenopausal women by traditional Chinese medicine practitioners," Menopause, vol. 7, no. 2, pp. 129-134, 2000. 


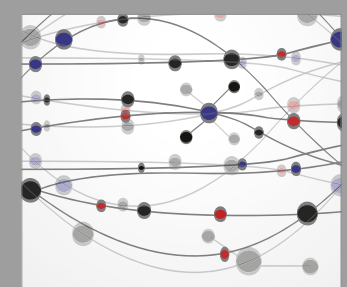

The Scientific World Journal
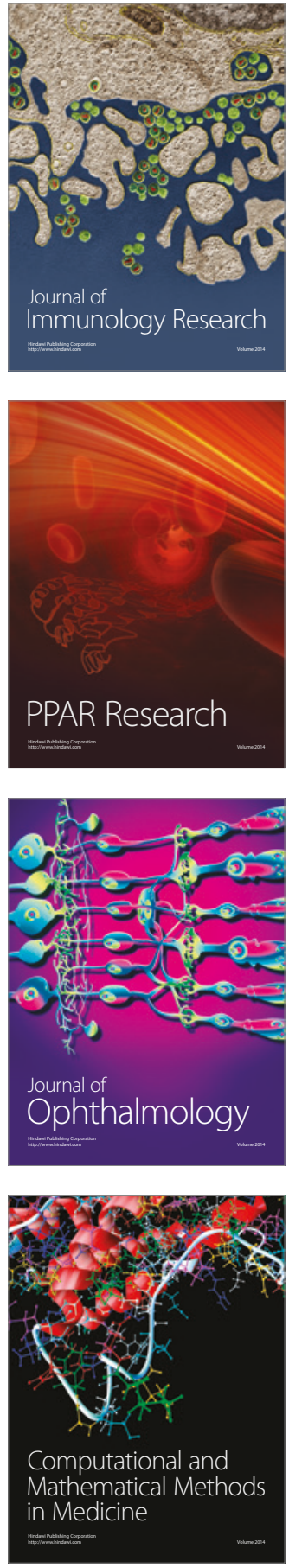

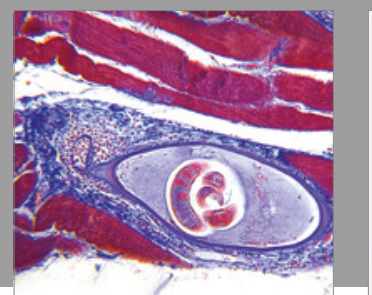

Gastroenterology Research and Practice
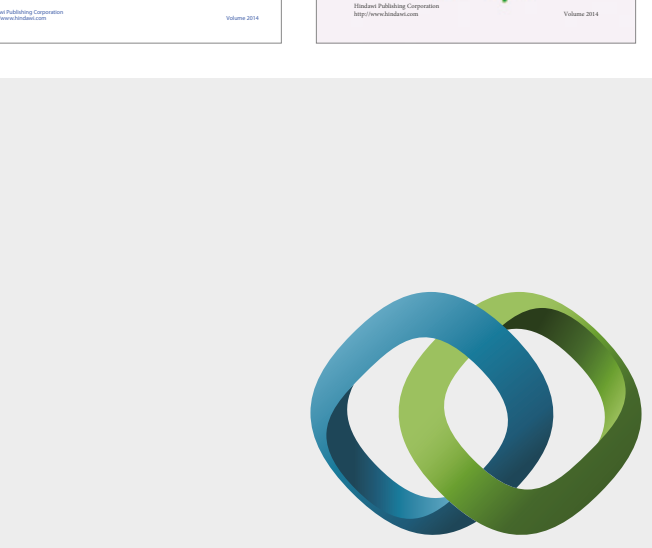

\section{Hindawi}

Submit your manuscripts at

https://www.hindawi.com
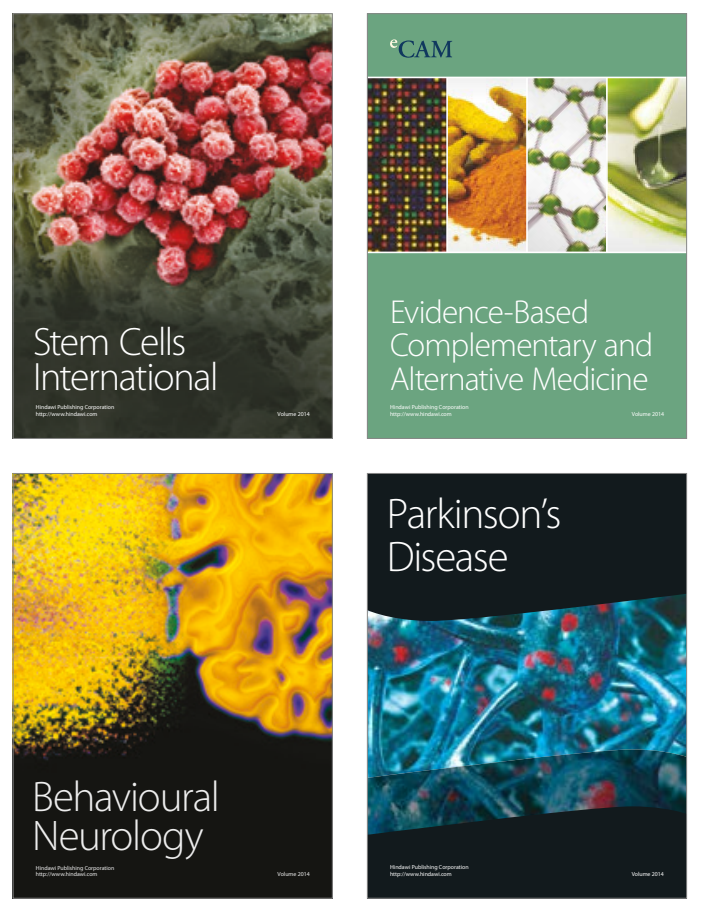
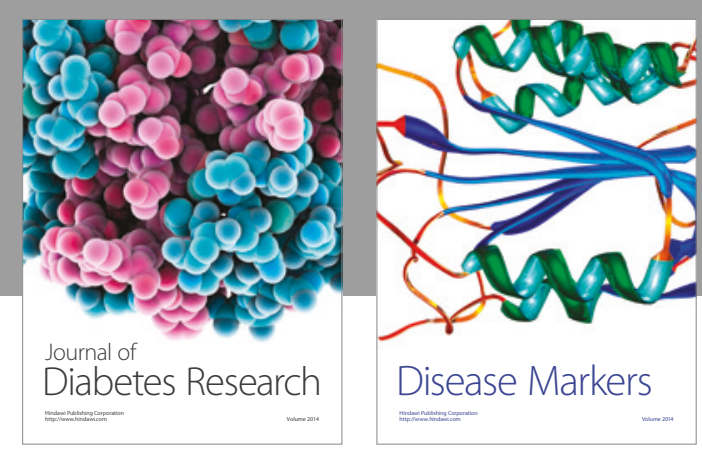

Disease Markers
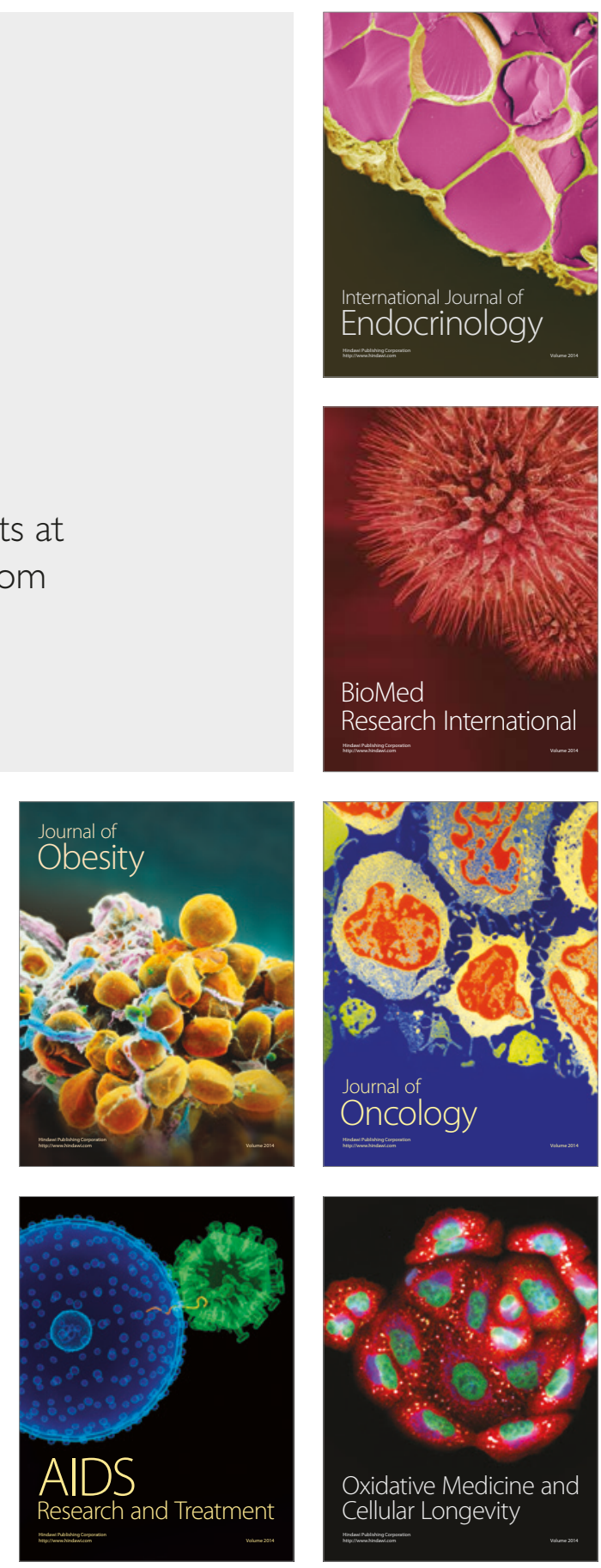